\title{
Motion Analysis of the Patella in Asymptomatic Patellofemoral Joints by Transparent 3D Imaging
}

\section{Masashi Mastuzaki ${ }^{*}$ and Norio Sekine}

Department of Radiological Science, Graduate School of Human Health Sciences, Tokyo Metropolitan University, Tokyo, Japan

*Corresponding author: Masashi Matsuzaki, Department of Radiological Science, Graduate School of Human Health Sciences, Tokyo Metropolitan University, Tokyo, Japan, Tel: 81-3-3815-1400; Fax: +81-3-3815-1408; E-mail: matsuzaki@sonic-j.com

Received date: November 20, 2017; Accepted date: November 24, 2017; Published date: November 27, 2017

Copyright: (c) 2017 Mastuzaki M, et al. This is an open-access article distributed under the terms of the Creative Commons Attribution License, which permits unrestricted use, distribution, and reproduction in any medium, provided the original author and source are credited.

\begin{abstract}
Transparent 3D images was used to analyze the motion of the patella in knee extension movements at the patellofemoral joint in asymptomatic persons aged 40 and over who are at increased risk of osteoarthritis of the knee.

By adding transparency to $3 \mathrm{D}$ images constructed by extracting the femoral condyle cartilage and patella from 3D data using MRI scans at knee flexion angles of $120^{\circ}, 90^{\circ}$ and $60^{\circ}$, the patella movement analysis images allow the position of the patella to be accurately ascertained through the joint cartilage. This made it possible to analyze the rotation and inclination of the patella when the patella is moved by the femoral trochlear during knee extension movements.

There was a correlation between patella rotation and patella inclination when the knee is extended. 3D motion analysis of the patella at the patellofemoral joint was performed by complex computer analysis according to conventional methods. Transparent 3D imaging facilitates the use of bony landmarks to analyze the motion of the patella. This method is thought to be useful in preventive medicine by enabling early prognostic prediction by performing motion analysis of the patella with respect to deformation of the patellofemoral joint.
\end{abstract}

Keywords: Patellofemoral joint; Transparent 3D imaging; Femoral trochlear; Patella motion

\section{Introduction}

Osteoarthritis of the knee is thought to be an irreversible progressive condition. Although people at risk of developing this condition are known to include the elderly, women, people with obesity, people with a history of knee injury, and people with a knee varus deformity, the underlying causes are still unknown [1-3]. Onset typically occurs after reaching 40 years of age, and it is clear that the prevalence increases sharply in people aged 50 and above [4-6]. The sites of cartilage degeneration in osteoarthritis of the knee are classified as medial condyle, lateral condyle and patellofemoral, with the latter occurring in $34 \%$ of females over the age of 60 according to the findings of X-ray observations [7]. Also, degeneration of the patellofemoral joint was found in $79 \%$ of cadavers aged 65 and over, showing that degeneration of the patellofemoral joint is very common [7]. Even at the early stages, MRI scans can reveal degeneration of cartilage in the trochlear groove inside the patellofemoral joint [8].

Most studies of patellofemoral joint disorders have analyzed the movement of the patella by means of simple X-ray, CT and MRI scans [9-18], but have mostly involved evaluations based on axial views, making it difficult to perform accurate patella motion analysis. Therefore, studies using cadavers are being performed in order to accurately analyze the movement of the patella [19-22]. However, these studies are also limited because cadavers do not move in the same way as living subjects. Recently, it has become possible to perform accurate evaluations of living subjects by means of computerized 3D motion analysis [23-25], but so far this method has mostly been used to analyze patella motion in patients with patellofemoral joint disorders having dislocated patellas, and few analyzed the motion of the patella in patients with osteoarthritis [26]. Furthermore, there have been no patella motion analysis studies of patients in the early stages of osteoarthritis of the knee. The aim of the present study was to examine the factors behind the early stages of osteoarthritis of the knee by using a new $3 \mathrm{D}$ imaging method to perform a motion analysis of the patella at the patellofemoral joint in asymptomatic subjects aged 40 and above, who are considered to be at risk of developing osteoarthritis of the knee.

\section{Methods}

The proposed technique was used to examine 13 knees of 13 asymptomatic volunteers ( 1 male, 12 female) from 44 to 58 years of age (average 50.6), who had all given their informed consent. The test subjects had no history of knee pain or trauma

3DMR imaging was performed using a 3-Tesla MR system (Intera Achieva 3.0 T Quasar Dual gradient R2.6; Philips, Best, The Netherlands) with a flex coil (Flex S_2 channel Phased Alley Coil; Philips, Best, The Netherlands) to obtain images at knee flexion angles of $120^{\circ}, 90^{\circ}$ and $60^{\circ}$. The limbs were examined in the lateral decubitus position, with care taken to ensure that the rotation of the tibia was not affected by the motion of the patella [20-22]. The MR scans were obtained by $3 \mathrm{D}$ WATS with a $15^{\circ}$ flip angle, $\mathrm{TR}=20 \mathrm{~ms}$, slice thickness $=1.5 \mathrm{~mm}$, and FOV $=150 \mathrm{~mm} \times 150 \mathrm{~mm}$.

The 3D images were constructed using Mimics Research 19.0 and 3matic Research 11.0 software (Materialise NV, Leuven, Belgium). The 
Page 2 of 6

3D images were constructed by extracting the femoral condyle cartilage and patella semi-automatically from 3D MR imaging data (Figure 1).

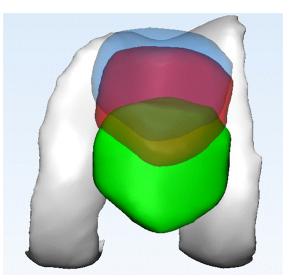

Figure 1: 3D image of the structure of the femoral condyle cartilage and patella.

Motion analysis of the patella in the patellofemoral joint was performed with regard to the movement, rotation and inclination of the patella in the femoral trochlear during knee extension. During movement of the patella, the positional relationship between the femoral trochlear area and patella was analyzed based on 3D images obtained with a 3-matic Research 11.0. First, the overall area of the femoral trochlear (trochlear total area; TTA) was determined by hiding the display of the patella from the $3 \mathrm{D}$ image (Figure $2 \mathrm{~A}$ ), and the patella was then displayed to determine the amount of the femoral trochlear not covered by the patella (trochlear show area; TSA) (Figure $2 \mathrm{~B})$. The TSA was divided by the TTA to yield the trochlear show area ratio (TSAR) and the TSAR angle at each knee flexion angle:

$\mathrm{TSAR}=\mathrm{TSA} / \mathrm{TTA} \times 100$

The mobility of the patella at different angles of knee flexion was obtained from the following formula:

Patella mobility between $120^{\circ}$ and $90^{\circ}$ TSAR $(120-90)=$ TSAR $120-$ TSAR90

Patella mobility between $90^{\circ}$ and $60^{\circ}$ TSAR (90-60) = TSAR90TSAR60

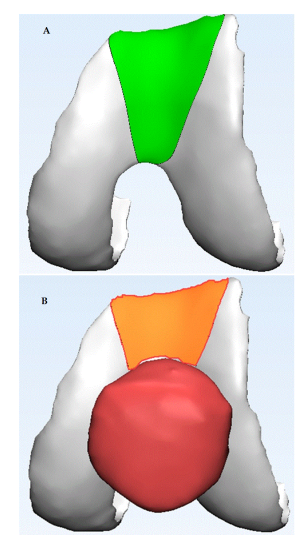

Figure 2: Femoral trochlear area ratio (TSAR90) analysis images. A. Total area of femoral trochlear (TTA90). B. Femoral trochlear display area (TSA90).

Using Mimics Research 19.0 software, the patella rotation angle was analyzed based on the femoral trochlear groove and the middle ridge of the patella in the 3D image. First, the viewpoint of the 3D image was aligned with a position from the back of the femoral condyle cartilage (Figure 3A).
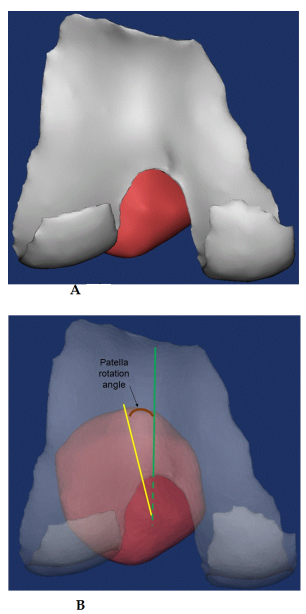

Figure 3: Patella rotation angle (PRA90) analysis image. A. Rear view of femoral condylar articular cartilage (transparency: 0) and patella (transparency: 0). B. Rear view of femoral condylar articular cartilage (transparency: high) and patella (transparency: medium).

To apply the new 3D imaging technique, the transparency was altered so that the joint cartilage and patella appeared transparent, and then the femoral trochlear groove line and patella middle ridge line were moved so as to align them vertically with the femoral trochlear groove line. The resulting view was imported as a JPEG image (Figure 3B).

By increasing the transparency of the femoral trochlea and reducing the transparency of the patella, it is possible to clarify the positional relationship between the femoral trochlea and the patella (which is situated at a deeper position). This makes it possible to analyze the movement of the patella on the femoral trochlea simply by using the characteristic bony landmarks of the femoral trochlea and patella.

Using ImageJ software (National Institutes of Health, Bethesda, $\mathrm{MD})$, this image was analyzed to determine the angle of intersection between the femoral trochlear groove line and patella middle ridge line (patella rotation angle; PRA). The change of patella rotation angle with knee flexion angle is obtained from the following formula:

Patella rotation angle between $120^{\circ}$ and $90^{\circ}$ PRA (12090) $=$ PRA120-PRA90

Patella rotation angle between $90^{\circ}$ and $60^{\circ}$ PRA (90-60) $=$ PRA90PRA60

The patella inclination angle is obtained using the patella tilt angle (PTA) and lateral facet angle (LFA) from the sky-line view used for evaluation by simple X-rays used to construct the 3D image $[27,28]$. The transparency was set so that the patella appears transparent, and the position was set so that the top and bottom ends of the line through the patella middle ridge were superimposed (Figure 4A). Lines were drawn between the bottom of the femoral medial condyle and the bottom of the lateral femoral condyle, and across the maximum lateral diameter of the patella, and the result was captured as a JPEG image (Figure 4B). This image was analyzed using ImageJ to find the angle of 
Citation: Mastuzaki M, Sekine N (2017) Motion Analysis of the Patella in Asymptomatic Patellofemoral Joints by Transparent 3D Imaging. J

Page 3 of 6

intersection between the two lines (PTA). The variation of PTA with the knee flexion angle was obtained from the following formula:

Patella tilt angle between $120^{\circ}$ and $90^{\circ}$ PTA $(120-90)=$ PTA120PTA90

Patella tilt angle between $90^{\circ}$ and $60^{\circ}$ PTA (90-60)=PTA90-PTA60

For the LFA, lines were drawn between the top of the femoral medial condyle and the top of the femoral lateral condyle, and across the lateral surface of the patella, and the result was captured as a JPEG image (Figure 4C). This image was analyzed using ImageJ to find the angle of intersection between the two lines (LFA). The variation of LFA with the knee flexion angle was obtained from the following formula:

Patella lateral facet angle between $120^{\circ}$ and $90^{\circ}$ LFA (12090)=LFA120-LFA90

Patella lateral facet angle between $90^{\circ}$ and $60^{\circ}$ LFA (90-60) $=$ LFA90LFA60

The patella motion was analyzed based on the relationships of PRA, PTA and LFA to the change in patella movement (TSAR) at different knee flexion angles while extending the knee.

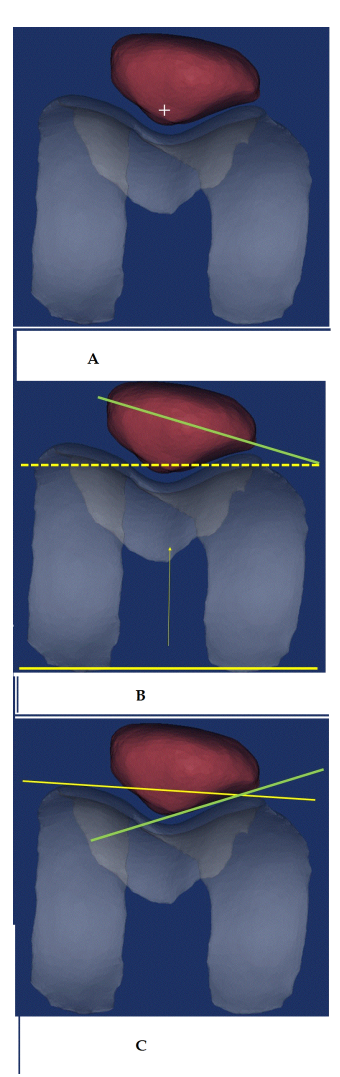

Figure 4: Patella inclination angle analysis image $\left(90^{\circ}\right.$ knee flexion). A. Sky line view 90 setting position. B. PTA (90). C. LFA (90).

When the knee was flexion at an angle of $60^{\circ}$, the sulcus angle was recorded as the angle between the lateral and medial surfaces of the femoral trochlear groove (Figure 5).

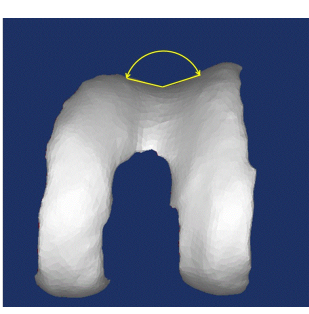

Figure 5: Sulcus angle analysis image.

\section{Results}

\section{Analysis of test subjects}

The age, gender, height, weight and sulcus angle of each of the thirteen test subjects are shown in Table 1 . None of the test subjects were excessively overweight. Furthermore, since all the test subjects had sulcus angles within the normal range, none of them had dysplasia of the patellofemoral joint $[9,29,30]$.

\begin{tabular}{|c|c|c|c|c|c|}
\hline Patients & Age (yrs) & Gender & $\begin{array}{l}\text { Height } \\
\text { (cm) }\end{array}$ & $\begin{array}{l}\text { Weight } \\
\text { (kg) }\end{array}$ & $\begin{array}{l}\text { Sulcus } \\
\text { angle }\left({ }^{\circ}\right)\end{array}$ \\
\hline & 55 & $F$ & 153 & 56 & 144 \\
\hline 2 & 47 & $\mathrm{~F}$ & 164 & 55 & 147 \\
\hline 3 & 55 & $\mathrm{~F}$ & 150 & 48 & 143 \\
\hline 4 & 55 & $M$ & 170 & 69 & 140 \\
\hline 5 & 53 & $\mathrm{~F}$ & 154 & 51 & 144 \\
\hline 6 & 49 & $\mathrm{~F}$ & 155 & 55 & 147 \\
\hline 7 & 47 & $\mathrm{~F}$ & 165 & 54 & 143 \\
\hline 8 & 50 & $F$ & 150 & 45 & 136 \\
\hline 9 & 50 & $F$ & 158 & 52 & 146 \\
\hline 10 & 46 & $\mathrm{~F}$ & 160 & 50 & 142 \\
\hline 11 & 49 & $F$ & 159 & 57 & 146 \\
\hline 12 & 44 & $\mathrm{~F}$ & 173 & 75 & 140 \\
\hline 13 & 58 & $F$ & 155 & 61 & 142 \\
\hline Mean & 50.6 & & 158.9 & 56 & 143.1 \\
\hline
\end{tabular}

Table 1: Clinical detail of the volunteers.

\section{Patella motion analysis}

1. TSRA and PRA in knee extension actions. The correlation between the patella mobility and patella rotation with respect to changes in the knee flexion angle when extending from $120^{\circ}$ to $90^{\circ}$ and from $90^{\circ}$ to $60^{\circ}$ was $\mathrm{r}=-0.77, \mathrm{P}=0.002$ between $120^{\circ}$ and $90^{\circ}$, and $\mathrm{r}=-0.76, \mathrm{P}=0.002$ between $90^{\circ}$ and $60^{\circ}$, indicating a strong negative relationship (Figures $6 \mathrm{~A}$ and $6 \mathrm{~B}$ ). 


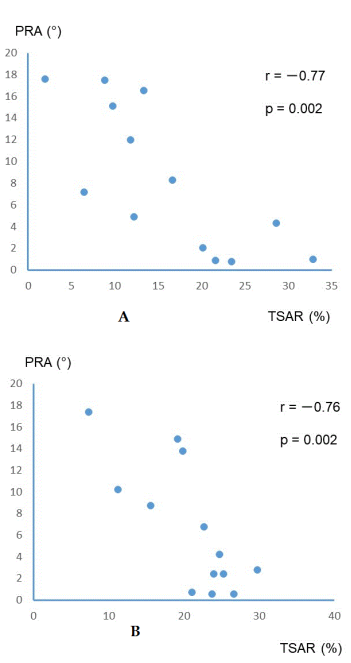

Figure 6: Correlation of Patella Rotation Angle (PRA) and Trochlear Show Area Ratio (TSAR). A. Knee flexion $120^{\circ}-90^{\circ}$. B. Knee flexion $90^{\circ}-60^{\circ}$.

1. TSRA and PTA in knee extension actions. The correlation between the patella mobility and patella PTA with respect to changes in the knee flexion angle when extending from $120^{\circ}$ to $90^{\circ}$ and from $90^{\circ}$ to $60^{\circ}$ was $\mathrm{r}=0.77, \mathrm{P}=0.002$ between $120^{\circ}$ and $90^{\circ}$, and $\mathrm{r}=0.72, \mathrm{P}=0.005$ between $90^{\circ}$ and $60^{\circ}$, indicating a strong positive relationship (Figures 7A-7C).

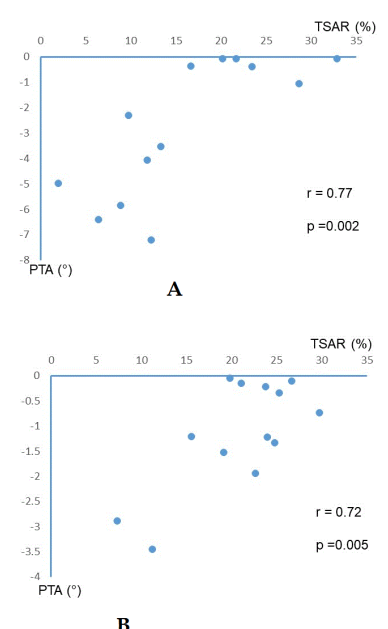

Figure 7: Correlation of Patella Tilt Angle (PTA) and Trochlear Show Area Ratio (TSAR). A. Knee flexion $120^{\circ}-90^{\circ}$. B. Knee flexion $90^{\circ}-60^{\circ}$. C. TSRA and LFA in knee extension actions.

The correlation between the patella mobility and patella LFA with respect to changes in the knee flexion angle when extending from $120^{\circ}$ to $90^{\circ}$ and from $90^{\circ}$ to $60^{\circ}$ was $\mathrm{r}=-0.83, \mathrm{P}<0.001$ between $120^{\circ}$ and $90^{\circ}$, indicating a strong negative relationship, and $\mathrm{r}=-0.57, \mathrm{P}=0.03$ between $90^{\circ}$ and $60^{\circ}$, indicating a weak negative relationship (Figures $8 \mathrm{~A}$ and $8 \mathrm{~B})$.

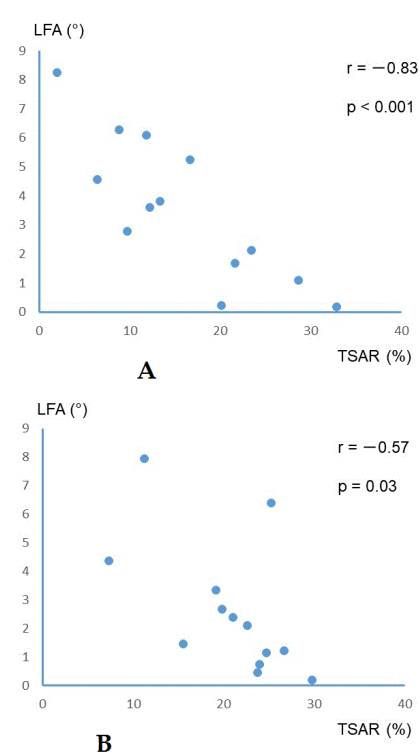

Figure 8: Correlation of Lateral Facet Angle (LFA) and Trochlear Show Area Ratio (TSAR). A. Knee flexion $120^{\circ}-90^{\circ}$. B. Knee flexion $90^{\circ}-60^{\circ}$.

\section{Discussion}

The new transparent 3D imaging method used in this study facilitates the evaluation of $3 \mathrm{D}$ patella movements at the patellofemoral joint, which has not been done before. This makes it easy to use 3D computer analysis algorithms to determine the shape of the patella and the patellofemoral joint in transparent 3D images showing the rotation and inclination of the patella in three dimensions [25]. Furthermore, with the method used in this study, MRI scans of the femoral condyle cartilage and patella can be used to perform accurate assessments of patella movement, which would hitherto have been performed by evaluation methods based on X-ray images and/or CT scans [31].

The patellofemoral joint showed a tendency for increased rotation and less inclination of the patella as the mobility of the patella at the femoral trochlear becomes smaller during knee extension actions. On the other hand, there was a weak correlation with LFA during knee extension movements from $90^{\circ}$ to $60^{\circ}$. This is thought to be attributable to the thickness of the patella cartilage. The adaptation of the patellofemoral joint starts from the $90^{\circ}$ knee flexion position at the articulating surfaces [32]. Up to $90^{\circ}$, a degree of freedom arises due to the thickness of the patella cartilage in the matching state where the articulating surfaces are the patella and the femoral trochlear groove (LFA90-60), and is considered to have a weak correlation. LFA120-90 shows a strong correlation with the movement of the patella, which is thought to be because with a larger knee flexion angle, the patella and femoral trochlear groove are matched together as a patellofemoral joint.

This analysis of patella motion in asymptomatic patellofemoral joints showed a significant correlation between the movement of the patella and the rotation and inclination of the patella when extending the knee joint. This is thought to reflect the function of the quadriceps muscle during knee extension. It has been reported that the functioning of the quadriceps muscle contributes to a high patella 
Page 5 of 6

position and degeneration of the patellofemoral joint [33]. Also, according to a report by Felicio et al., the patella reflects the function of the quadriceps femoris muscle, and the contraction of the quadriceps muscle and PTA are closely related [34]. Osteoarthritis of the knee is closely related to the function of the quadriceps muscle, and the finding of the present study suggests that the patella's movement, inclination and rotation reflect the latent function of the quadriceps muscle.

Based on the results of this study, the small amount of movement by the patella means that the patella rotates and its inclination decreases. In other words, the patella continues to press against the inner side of the femoral trochlear groove. Studies performed using cadavers have reported that in people with osteoarthritis of the knee, the patellofemoral joint cause's strong degeneration of cartilage in the femoral trochlear groove [35-39]. Although the patellofemoral joint is not intrinsically weight-bearing, it is said that a large pressure (up to five times the body weight) is applied to the joint surface during knee flexion and extension movements, especially when walking up and down stairs $[40,41]$. Our research suggests that one of the causative factors in the early stages of osteoarthritis of the knee is the deterioration of patella movement with declining function of the quadriceps femoris muscle, bringing the patella into stronger contact with the femoral condyle cartilage of trochlear and leading to the onset of degeneration during everyday activities.

In this study, since e the latent function of the quadriceps femoris muscle was evaluated statically, it was not possible to evaluate dynamic movements during extension and flexion, or dynamic changes in the load on the patella. High-resolution MRI scans are limited in terms of their ability to perform evaluations in conjunction with the movement of joints. On the other hand, ultrasonography has become widely used in the study of musculoskeletal disorders due to its ability to produce high-resolution scans of superficial regions [42]. According to Imai et al. the medial patellar retinaculum makes a large contribution to movement of the patella during extension and flexion of the knee [43]. In anatomical studies of cadavers, the medial patellar femoral ligament, which is a constituent of the medial patellar retinaculum, could be visualized by ultrasonography [44]. It is expected that the degeneration of patellofemoral joints could be evaluated by using ultrasonography to assess the rotation and inclination of the patella while dynamically observing the tone of the medial patellar femoral ligament. Using ultrasonography, it should be possible to analyze the movement of the patella by performing dynamic evaluations in real time with high resolution.

\section{Conclusion}

In summary, a study of the early stages of degeneration of patellofemoral joints in asymptomatic subjects has been performed by using transparent 3D images to analyze the motion of the patella. This should result in improved preventive medicine by making it easier to detect risk factors and impede the progress of osteoarthritis.

\section{Ethical Statements}

All procedures followed were in accordance with the ethical standards of the responsible committee on human experimentation (institutional and national) and with the Helsinki Declaration of 1964 and later versions. Informed consent was obtained from all patients for being included in the study.

\section{Conflict of Interest}

The authors declare that there is no conflict of interest.

\section{References}

1. Hrat DJ, Doyle DV, Spector TD (1999) Incidence and risk factors for radiographic knee osteoarthritis in middle-aged women: the Chingford Study. Arthritis Rheum 42: 17-24.

2. Brouwer GM, Van Tol AW, Bergink AP, Belo JN, Bernsen RM, et al. (2007) Association between valgus alignment and the development and progression of radiographic osteoarthritis of the knee. Arthritis Rheum 56: 1204-1211.

3. Sudo A, Miyamoto N, Horikawa K, Urawa M, Yamakawa T, et al. (2008) Prevalence and risk factors for knee osteoarthritis in elderly Japanese men and women. J Orthop Sci 13: 413-418.

4. Yoshimura N, Muraki S, Oka H, Mabuchi A, En-Yo Y, et al. (2009) Prevalence of knee osteoarthritis, lumbar spondylosis and osteoporosis in Japanese men and women: The Research on Osteoarthritis/osteoporosis Against Disability (ROAD). J Bone Miner Metab 27: 620-628.

5. Muraki S, Oka H, Akune T, Mabuchi A, En-yo Y, et al. (2009) Prevalence of radiographic knee osteoarthritis and its association with knee pain in the elderly of Japanese population-based cohorts: The ROAD study. Osteoarthritis Cartilage 17: 1137-1143.

6. Yoshimura N, Muraki S, Oka H, Kawaguchi H, Nakamura K, et al. (2010) Cohort Profile: Research on Osteoarthritis/osteoporosis Against Disability (ROAD) Study. Int J Epidemiol 39: 988-995.

7. Noble J, Hamblen DL (1975) The pathology of the degenerate meniscus lesion. J Bone Joint Surg Br 57: 180-186.

8. Hada S, Kaneko S, Sadatsuki R, Liu L, Futami I, et al. (2014) The degeneration and destruction of femoral articular cartilage shows a greater degree of deterioration than that of the tibial and patellar articular cartilage in early stage knee osteoarthritis: a cross-sectional study. Osteoarthr Cartil 22: 1583-1589.

9. Merchant AC, Mercer RL, Jacobset RH, Cool CR (1974) Roentgenographic analysis of patellafemoral congruence. J Bone Joint Surg 56: 1391-1396.

10. Schutzer SF, Ramby GR, Fulkerson JP (1986) The evaluation of the patellofemoral pain using computerized tomography. Clin Orthop 204: 286-293.

11. Stanford W, Phelan J, Kathol MH, Rooholamini SA, El-Khoury GY, et al. (1988) Patellofemoral joint motion: Evaluation by ultrafast computed tomography. Skeletal Radiol 17: 487-492.

12. Pinar H, Akseki D, Karaogland O, Genc I (1994) Kinematic and dynamic axial computed tomography of the patella-femoral joint in patien patients with anterior knee pain. Knee Surg Sports Trauma Arthosc 2: 170-173.

13. Shellock FG, Mink JH, Fox JM (1988) Patellofemoral joint: Kinematic MR imaging to assess tracking abnormalities. Radiology 168: 551-553.

14. Brossman J, Muhle C, Schroder C, Melchert UH, Büll CC, et al. (1993) Patellar tracking pattern during active and passive knee extension: Evaluation with motion-trigger cine MR imaging. Radiology 206: 205-212.

15. Sheehan FT, Zajac FE, Drance JE (1999) In vivo tracking of the human patella using cine phase contrast magnetic resonance imaging. J Biomech Eng 121: 650-656.

16. Muhle C, Brossman J, Heller M (1999) Kinematic CT and MR imaging of the patellofemoral joint. Eur Radiol 9: 508-518.

17. Hraman M, Dogan A, Arslan H, Ipeksoy U, Vural S (2002) Evaluation of the patellofemoral joint with kinematic MR fluoroscopy. Clin Imaging 26: 136-139.

18. O Donnell P, Johnstone C, Watson M, McNally E, Ostlere S (2005) Evaluation of patellar tracking in symptomatic and asymptomatic individual by magnetic resonance imaging. Skeletal Radiol 34: 130-135.

19. Fujikawa K, Seedhom BB, Wright V (1983) Biomechanics of the patellofemoral joint. Part 1: a study of the contact and the congruity of the 
Citation: Mastuzaki M, Sekine N (2017) Motion Analysis of the Patella in Asymptomatic Patellofemoral Joints by Transparent 3D Imaging. J

Page 6 of 6

patello-femoral compartment and movement of the patella. Eng Med 12: 3-11.

20. Hefzy MS, Jackson WT, Saddemi SR, Hsieh YF (1992) Effect of tibial rotations on patellar tracking and patello-femoral contact areas. J Biomed Eng 14: 329-343.

21. Heegaard J, Leyvaz PF, Van Kampen A, Rakotomanana L, Rubin PJ, et al. (1994) Influence of soft structures on patella three-dimensional tracking. Clin Orthop 299: 235-243.

22. Heegaard J, Leyvaz PF, Curnier A, Rakotomanana L, Huiskes R (1995) The biomechanics of the human patella during passive knee flexion. J Biomech 28: 1265-1279.

23. Von Eisenhart-Rothe R, Siebert M, Bringmann C, Vogl T, Englmeier KH, et al. (2004) A new in vivo technique for determination of 3D kinematics and contact areas of the patella-femoral and tibio-femoral joint. J Biomech 34: 927-934.

24. Fellows RA, Hill NA, Gill HS, MacIntyre NJ, Harrison MM, et al. (2005) Magnetic resonance imaging for in vivo assessment of three-dimensional patella tracking. J Biomech 38: 1643-1652.

25. Yamada Y, Toritsuka Y, Shino K, Sugamoto K, Yoshikawa H, et al. (2007) In vivo movement analysis of the patella using a three-dimensional computer model. J Bone Joint Surg Br 89: 752-760.

26. McWalter EJ, Hunter DJ, Harvey WF, McCree P, Hirko KA, et al. (2011) The effect of a patellar brace on three-dimensional patellar kinematic in patients with lateral patellofemoral osteoarthritis. Osteoarthritis Cartilage 19: 801-808.

27. Merchant CA (2001) Patellofemoral imaging. Cli Orthop Relat Res 389: $15-21$.

28. Laurin CA, Dussalt R, Levesque HP (1979) The tangential $\mathrm{x}$-ray investigation of the patellofemoral joint: $\mathrm{x}$-ray technique, diagnostic criteria and their interpretation. Clin Orthop Relat Res 144: 16-26.

29. Brattstroem H (1964) Shape of the intercondylar groove normally and in recurrent dislocation of patella: a clinical and $\mathrm{x}$-ray-anatomical investigation. Act Orthop Scand Suppl 68: 1-148.

30. Nicolas L, Tigchelaar S, Koëter S (2011) Patellofemoral evaluation with magnetic resonance imaging in 51 knee of asymptomatic subject. Knee Surg Sports Trauma Arthosc 19: 1735-1739.

31. Staubli HU, Dürrenmatt U, Porcellini B, Rauschning W (1999) Anatomy and surface geometry of patellofemoral joint in the axial plane. J Bone Joint Surg Br 81: 452-458
32. Davies-Tuck M, Teichtahl AJ, Wluka AE, Wang Y, Urquhart DM, et al. (2008) Femoral sulcus angle and increased patella facet cartilage volume in an osteoarthritic population. Osteoarth Cartil 16: 131-135.

33. Joshua J, Guermazi A, Zhu Y, Zumwalt AC, Gross KD, et al. (2011) Quadriceps weakness, patella alta and structural features of patellofemoral osteoarthritis: The multicenter osteoarthritis study. Arthritis Care Res 63: 1391-1397.

34. Felicio LR, Saad MC, Liporaci RF, Baffa Ado P, Dos Santos AC, et al. (2010) Evaluation patellar kinematic through magnetic resonance imaging during open and closed kinetic chain exercise. J Sport Rehabili 19: 1-11.

35. Owre A (1936) chondromalacia patella. Acta Chir Scand.

36. Helfet AJ (1974) Disorder of the knee. Lippincott Comp, Philadelphia, USA.

37. Meachim G, Emery HI (1974) Quantitative aspect of patella-femoral cartilage fibrillation in Liverpool necropsies. Ann Rheum Dis 33: 39-47.

38. Insall J (1976) Chondromalacia patellae. J Bone and Joint Surg 58: 1-8.

39. Casscells SW (1978) Gross pathological changes in the knee joint of the age individual. Clin Orthop Relat Res 132: 225-232.

40. Li G, Papannagari R, Nha KW, Defrate LE, Gill TJ, et al. (2007) The coupled motion of the femur and patella during in vivo weightbearing knee flection. J Biomech Eng 129: 937-943.

41. Suzuki T, Hosseini A, Li JS, Gill TJ 4th, Li G (2012) In vivo patellar tracking and patella femoral cartilage during dynamic stair ascending. J Biomech 45: 2432-2437.

42. Matsuzaki M (2017) The latest technology of musculoskeletal ultrasonography: iterative revolution. J Med Ultrasonics 44: 223-226.

43. Imai N, Tomatsu T, Nakaseko J, Terada H (1987) Clinical roentgenological studies on malalignment disorder of the patello-femoral joint. Part 2: Relationship between predisposing factor and malalignment of the patello-femoral joint. Nippon Seikeigeka Zasshi 61: 1191-1202.

44. Phornphutkul C, Sekiya JK, Wojtys EM, Jacobson JA (2007) Sonographic Imaging of the patellofemoral medial joint stabilizing structures: Finding in human cadavers. Orthopedics 30: 472-478. 